\title{
Intérêt de l'extraction en phase solide en toxicologie : exemple d'extraction de 15 substances toxiques et médicamenteuses par 7 colonnes SPE différentes par un protocole unique
}

\section{Interest of SPE in toxicology field : example of extraction of 15 toxics or drugs extraction by 7 different SPE columns using a simple extraction procedure}

Nouredine SADEG*, Michel DUMONTET

(1) Laboratoire Claude Bernard, Centre Hospitalier René Dubos - F-95300 PONTOISE - France

*Auteur à qui adresser la correspondance : Nouredine SADEG, Laboratoire Claude Bernard, Centre Hospitalier René Dubos - F-95300 PONTOISE - France Tél int 33130754254 - Fax int +3313075 5369 - e-mail : sadeg @ch-pontoise.fr

(Reçu le 18 octobre 2000 ; accepté le 20 décembre 2000)

\section{RÉSUMÉ}

Nous voyons apparaître sur le marché de nouvelles colonnes d'extraction en phase solide avec de nouvelles matrices dédiées pour l'extraction, en une seule étape, de toxiques divers et les fabricants proposent pour ce faire, des protocoles spécifiques pour le conditionnement des colonnes, le choix des tampons, les phases de lavage et d'élution des toxiques à extraire. Nous avons utilisé un protocole unique pour l'extraction en une seule étape de médicaments acides, neutres et basiques. Un protocole d'extraction en général est un compromis entré la sélectivité et le taux de rendement. Seul le taux de rendement est déterminé dans notre étude. Le protocole d'extraction utilisé est le suivant : conditionnement par méthanol et tampon phosphate pH 4,4, passage de l'échantillon, lavage par la solution tampon $\mathrm{pH}$ 4,4 et élution par le méthanol. La colonne OASIS extrait correctement les différents médicaments analysés. Aux conditions utilisées, les

\section{SUMMARY}

We see to appear of new SPE columns dedicated for the extraction, in one stage, of various poisonous and proposes for it make, some specific protocols for the conditioning of the columns, the choice of the buffers, solvents for washing and eluting steps. We used an unique protocol for the extraction in one stage of acidic, neutral and basic drugs and toxics. A protocol of extraction generally is a compromise between the selectivity and the rate of recovery. Only the rate of recovery is determined in our study. The protocol of used extraction is the following: conditioning by methanol and $\mathrm{pH}$ 4.4 phosphate buffer, pouring the sample, washing with $\mathrm{pH}$ 4.4 buffer solution and eluting the analytes. with methanol. The OASIS column extracts the different analyzed drugs correctly. To the used parameters, the Certify, MN Drugs Isolute HCX and Speed Flow ABN columns extract the basic and neutral molecules weakly. Indeed, the basic analytes are not 
colonnes Certify, MN Drugs Isolute HCX et Speed Flow ABN extraient faiblement les molécules basiques et neutres. En effet, les analytes basiques ne sont pas élués et restent accrochés à la matrice ; il faudra, pour augmenter la force éluante, alcaliniser l'éluat par du $\mathrm{NH}_{4} \mathrm{OH}$ par exemple. Ceci permettra de créer un recul d'ionisation et donc de décrocher ces analytes de la phase stationnaire. Ces médicaments basiques sont donc correctement élués lorsque l'on rajoute de l'ammoniac au solvant d'élution (méthanol). Par contre, les médicaments acides sont faiblement extraits car peu retenus par la phase stationnaire. Pour améliorer le rendement d'extraction par ces colonnes, il faudra modifier les conditions de l'étape de lavage selon les molécules à extraire. En effet, le mécanisme de rétention est fonction du choix des tampons et de leurs $p H$. La colonne MNHR-P extrait seulement les médicaments acides. La colonne Isolute HAX est intéressante par ses performances, mais donne de faibles taux de rendement pour le méthotrexate et les salicylés. Les colonnes OASIS, Speed Flow ABN et MN HR-P sont indiquées pour l'extraction sur sang total, ce qui n'est pas le cas pour les autres colonnes étudiées et ceci est une donnée importante en Toxicologie médico-légale.

\section{MOTS-CLÉS}

Colonnes SPE, toxique, médicament, extraction sur phase solide.

\section{Introduction}

La recherche toxicologique pour la détermination des molécules impliquées dans l'intoxication est une approche analytique de plus en plus adoptée par plusieurs laboratoires hospitaliers (1). Plusieurs études ont été publiées portant sur différentes techniques de séparation et de détection (GC-MS (2), HPLC-DAD (3-4), TLC (5)). L'utilisation des méthodes d'extraction sur phase solide (SPE) pour isoler en une seule fois plusieurs toxiques et leurs métabolites est récente, ce qui explique le peu d'études comparant ces méthodes entre-elles et avec les méthodes d'extraction conventionnelles liquide-liquide.

L'objet de cette étude est de montrer l'intérêt en recherche toxicologique des méthodes d'extraction en phase solide (SPE), à côté des techniques d'extraction liquide-liquide.

\section{Méthodes}

Les colonnes SPE étudiées sont : colonnes Isolute $\mathrm{HCX}$ et Iśolute Hax (IST, MID Glamorgan, UK) ; colonnes MN Drugs et MN HR-P (Macherey Nagel, Düren, Allemagne) ; colonne Certify (Varian, harbor City, USA) ; colonne OASIS (Waters, Milford, MA) ; et colonne Speed Flow ABN (Applied Separation, Allentown, PA).

Les médicaments testés sont de structure chimique dif- eluted and remain hung to the matrix; it will be necessary, in order to increase the strength of elution, to alkalize the eluting solvent with $\mathrm{NH}_{4} \mathrm{OH}$ for example and this will allow to fall back ionization and therefore of unhooking these analytes from the stationary phase. Therefore, these basic drugs are correctly eluted when the ammonia is added to the elution solvent (methanol). On the other hand, the acidic drugs are weakly extracted because of little kept by the stationary phase. In order to improve the output of extraction by these columns, it will be necessary to modify the step of washing according to molecules to extract. Indeed, the mechanism of retention is function of choice of buffers and of their $p H$. The MNHR-P column extracts the acidic drugs only. The Isolute HAX column is interesting by its performances, but deal of weak rate of recovery for methotrexate and salicylate. The OASIS, Speed Flow ABN and MNHR-P columns are indicated for the extraction on whole blood which is not the case for the other studied columns and this is an important data in forensic toxicology.

\section{KEY-WORDS}

SPE column, toxic, drug, solid-phase extraction.

férente avec des propriétés physico-chimiques diverses. Cette étude inclue - entre parenthèses, les concentrations respectives finales testées - : (a) molécules avec pKa acide : paracétamol $(121 \mathrm{mg} / \mathrm{l})$, acide salicylique $(24,5 \mathrm{mg} / \mathrm{l})$, acide valproïque $(120 \mathrm{mg} / \mathrm{l})$, méprobamate $(50 \mathrm{mg} / \mathrm{l})$, et phénobarbital $(37 \mathrm{mg} / \mathrm{l})$; (b) molécules avec pKa neutre ou faiblement basique : théophylline $(27 \mathrm{mg} / \mathrm{l})$, méthotréxate $(4 \mathrm{mg} / \mathrm{l})$, diazépam $(1 \mathrm{mg} / \mathrm{l})$, carbamazépine $(16 \mathrm{mg} / \mathrm{l})$, phénytoöne $(24,5 \mathrm{mg} / \mathrm{l})$, et digitoxine $(39 \mu \mathrm{g} / \mathrm{l})$; et (c) molécules avec pKa basique: amphétamine $(3 \mu \mathrm{g} / \mathrm{l})$, imipramine $(5 \mu \mathrm{g} / \mathrm{l})$, morphine $(2 \mu \mathrm{g} / \mathrm{l})$, et hydrocodone $(2 \mu \mathrm{g} / \mathrm{l})$. Ces produits sont présentés sous forme de solutions distribuées par Bio-Rad (Hercules, CA) et Asqualab (Issy les Moulineaux, France).

Les solutions de travail sont préparées en diluant les solutions mères dans du sérum humain mais aussi dans du sang total humain hépariné pour tester les performances de ces colonnes SPE en vue d'extraire des toxiques à partir d'une matrice sang total.

Tous les réactifs sont de qualité analytique et proviennent des Laboratoires Merck (Darmstatdt, Allemagne). Les analyses ont été effectuées selon les techniques suivantes:

Automate CLHP (Remedi, Bio-Rad) : Le Remedi utilise un système d'identification à balayage UV avec une séparation par chromatographie liquide de haute performance multicolonne (6). Morphine, hydrocodone, 
amphétamine, diazépam, et imipramine ont été analysées par cette technique. Le Remedi utilise une extraction on-line. Les taux de rendements ne sont donc pas affectés par cette technique car les solutions standards sont analysées de la même façon. Le calcul des concentrations (hauteurs de pics) se fait par comparaison à des solutions titrées.

FPIA : paracétamol, carbamazépine, digitoxine, méthotréxate, phénobarbital, phénytoüne, théophylline et acide valproïque ont été analysés par FPIA (7) sur l'analyseur FLx (Abbott, Park, IL) selon les instructions du fabricant.

TLC : La chromatographie planaire a été utilisée selon les méthodes déjà largement utilisées pour la détection des médicaments (8). Le solvant d'élution est un mélange chloroforme : acétone : diéthylamine : (50 $: 40: 10 \mathrm{v}: \mathrm{v}: \mathrm{v})$ pour le méprobamate. Le solvant de révélation du méprobamate est un mélange à parties égales de diphenylcarbazone $(0.5 \mathrm{~g} / \mathrm{l}$ dans le dichlorométhane) et de chlorure mercurique $20 \mathrm{~g} / \mathrm{l}$ dans l'éthanol.

La réaction de Trinder a servi pour quantifier les salicylés en spectrométrie UV (9).

Extraction : Le protocole d'extraction est le même pour toutes les colonnes : la colonne est conditionnée par un volume de méthanol suivi par un volume de tampon phosphate acide $\mathrm{KH}_{2} \mathrm{PO}_{4} 2,11 \%$ (poids/vol) pH 4,4. $2 \mathrm{ml}$ de ce tampon phosphate isotonique sont ajoutés à $1 \mathrm{ml}$ de sérum ou de sang total hepariné. Après avoir agité au Vortex ce mélange (pH 5,2) est transvasé dans la colonne. La colonne est lavée par 2 volumes de tampon phosphate, puis séchée 5 minutes. Enfin, l'échantillon est élué par 2 fois $0,5 \mathrm{ml}$ de méthanol. L'extrait est évaporé sous courant d'azote à $40^{\circ} \mathrm{C}$. Le résidu est repris par $1 \mathrm{ml}$ de tampon FLx (Abbott). L'avantage de ce tampon FLx est sa capacité à dissoudre toutes les substances choisies dans notre étude (résultats personnels, non publiés).

Le taux de rendement est calculé selon le rapport du taux déterminé de l'analyte dosé par FPIA ou Remedi après extraction sur le taux du même analyte déterminé par FPIA ou Remedi avant extraction. Ce rapport est multiplié par 100 pour l'exprimer en pourcentage. Les concentrations testées pour chaque produit sont les concentrations annoncées ci-dessus.

\section{Résultats}

Les méthodes analytiques que nous avons utilisé (Remedi, FPIA, TLC, réaction de Trinder) ne sont pas adéquates pour comparer avec une grande exactitude les différentes colonnes d'extraction. Or, il ne s'agit absolument pas d'un travail de comparaison. Néanmoins, ces méthodes permettent de rendre compte de l'intérêt des techniques SPE en Toxicologie analytique. Car, il faut bien comprendre que pour mener un travail de comparaison des différentes colonnes SPE, il aurait fallu optimiser chaque méthode d'extraction avec chaque type de colonne SPE pour chaque famille chimique médicamenteuse.

L'impact des deux éventuels biais (procédure d'extraction et méthodes analytiques utilisées) est minime pour l'objectif assigné à cette étude à savoir montrer l'intérêt de la SPE en toxicologie analytique par l'étude de différentes colonnes SPE.

Le tableau 1 résume les taux de recouvrements des différentes colonnes SPE pour les diverses substances étudiées dans le sérum sauf quelques cas particuliers (phénobarbital pour les 3 colonnes et le paracétamol pour Certify). Le taux de rendement d'extraction pour le méprobamate n'a pas pu être calculée car la chromatographie planaire (TLC) utilisée était qualitative.

Les colonnes Certify, MN Drugs et Isolute $\mathrm{HCx}$ extraient correctement les médicaments faiblement basiques et neutres. Le taux de rendement pour les médicaments acides est par contre très faible. En fait, ces analytes acides sont faiblement retenus par ces colonnes. Par contre les médicaments basiques sont fortement retenus par ces colonnes et le solvant d'élution utilisé dans ce protocole (méthanol) n'a pas une force d'élution suffisante pour décrocher ces médicaments de ces colonnes. Pour améliorer le taux de rendement de ces 3 colonnes pour les médicaments basiques, il suffit d'augmenter la force d'élution du solvant en rajoutant par exemple de l'ammoniac au solvant d'élution (en général on prépare en extemporané un mélange méthanol/ammoniac avec une teneur finale d'ammoniac à $2 \%$ exprimée en volume). Il est à souligner que dans notre étude le solvant d'élution est constitué seulement de méthanol.

La colonne MN HR-P est non adéquate pour une recherche toxicologique de substances médicamenteuses basiques. Ceci était prévisible, car c'est une colonne dédiée pour l'extraction de produits fortement acides comme les pesticides. Or, les médicaments acides sont en fait des acides faibles avec des pKa aux alentours de 4 et ceci explique les mauvais rendements de la théophylline et du méprobamate obtenus pour cette colonne MN HR-P.

La colonne Speed Flow ABN donne des taux de rendements faibles pour l'acide valproïque, l'imipramine, l'hydrocodone et les salicylés. Par contre, les taux de 
Tableau I : Taux de rendements d'extraction par différentes colonnes SPE de diverses substances chimiques médicamenteuses à partir du sérum (pour chaque extraction, $n=3$ ). Pour le protocole d'extraction, voir le paragraphe méthodes dans le texte.

\begin{tabular}{|lccccccc|}
\hline & Certify & MN Drugs & MN HR-P & Oasis & Isolute & Isolute & Speed \\
& \% & $\%$ & \% & \% & HCx \% & Hax \% & Flow \% \\
\hline Paracétamol & 97 & 51 & 62 & 121 & 47 & 65 & 39 \\
Carbamazépine & 109 & 114 & 19 & 113 & 100 & 106 & 75 \\
Digitoxine & 100 & 99 & 5 & 86 & 71 & 85 & 56 \\
Méthotréxate & 100 & 100 & 100 & 100 & 100 & 2,5 & 100 \\
Phénobarbital & 105 & 110 & 43 & 110 & 102 & 110 & 78 \\
Phénytoïne & 109 & 99 & 41 & 77 & 92 & 111 & 69 \\
Méprobamate & ND* & ND & ND & détecté & détecté & détecté & détecté \\
Salicylés & 0 & 0 & 65 & 58 & 0 & 23 & 0 \\
Théophylline & 97 & 89 & 16 & 106 & 95 & 102 & 72 \\
Valproate & 55 & 55 & 39 & 36 & 62 & 74 & 8 \\
Diazépam & 46 & 57 & 15 & 44 & 54 & 55 & 58 \\
Amphétamine & 9 & 0 & 18 & 80 & 45 & 68 & 76 \\
Imipramine & 0 & 0 & 3 & 91 & 7 & 99 & 17 \\
Morphine & 0 & 0 & 22 & 77 & 19 & 71 & 36 \\
Hydrocodone & 0 & 0 & 14 & 91 & 0 & 61 & 7 \\
\hline
\end{tabular}

$* \mathrm{ND}=$ non détecté.

rendements sont corrects pour les autres médicaments.

La colonne Isolute HAx semble intéressante, mais présente de faibles taux de rendements pour les salicylés et le méthotréxate.

La colonne OASIS semble être la colonne de choix pour le protocole d'extraction choisi et permet d'extraire en une seule fois un large éventail de médicaments acides, neutres et basiques. Ceci ne veut pas dire que la colonne OASIS soit meilleure que les autres colonnes, car les taux de rendements peuvent être meilleurs pour une autre colonne si l'on veut extraire spécifiquement une famille chimique avec un protocole d'extraction bien défini. Cette étude permet seulement de situer les limites et intérêts de toutes ces colonnes.

Le tableau 2 montre des taux de rendements d'extractions à partir du sang total pour les colonnes qui ont montré de bons rendements d'extraction à partir de la matrice sérique. Nous pouvons remarquer que les taux de rendements sur sang total sont similaires à ceux trouvés dans le sérum. Cependant, seules 3 colonnes (OASIS, Speed Flow et MN HR-P) permettent des extraits non hématiques (taux d'hémoglobine inférieur à $0,1 \mathrm{~g} / \mathrm{dl}$, déterminé par le Co-Oximeter de IL, Milan, Italy).

Tableau II : Taux de rendements d'extraction par différentes colonnes SPE de diverses substances chimiques médicamenteuses à partir du sang total (pour chaque extraction, $n=3$ ). Pour le protocole d'extraction, voir le paragraphe méthodes dans le texte.

\begin{tabular}{|lccccccc|}
\hline & Certify & MN Drugs & MN HR-P & Oasis & .Isolute & Isolute & Speed \\
& \% & \% & \% & \% & HCx \% & Hax \% & Flow \% \\
\hline Théophyline & 101 & 78 & 8 & 91 & 74 & 93 & 66 \\
Carbamazépine & 105 & 98 & 13 & 95 & 93 & 101 & 97 \\
Digitoxine & 93 & 85 & 9 & 103 & 73 & 95 & 82 \\
Valproate & 62 & 72 & 31 & 80 & 35 & 58 & 55 \\
Phénobarbital & 112 & 103 & 30 & 97 & 96 & 105 & 96 \\
Phénytoïne & NT* & 109 & 33 & 102 & 100 & 107 & 104 \\
\hline
\end{tabular}

${ }^{*} \mathrm{NT}=$ non testé. 


\section{Discussion}

Les toxicologues analystes ont toujours préféré les techniques d'extraction liquide-liquide, et ce, parce que ce sont des techniques qui ont déjà fait leurs preuves et permettent même de traiter des prélèvements délicats comme des liquides biologiques visqueux ou des prélèvements putréfiés. Ainsi, la technique SPE montrait des limites par rapport aux techniques d'extraction liquideliquide à savoir : (1) l'extraction est ciblée pour une molécule donnée avec ou sans ses congénères et donc non utilisable pour l'extraction large de différentes familles chimiques, (2) la répétabilité inter-lots des colonnes SPE était médiocre et par conséquent les taux de rendements obtenus étaient loin d'être reproductibles, (3) l'impossibilité de pouvoir travailler sur toute la gamme de $\mathrm{pH},(4)$ et enfin la capacité d'extraction des matrices à silice était limitée ; or en toxicologie, on rencontre souvent des surdosages et donc on peut craindre de dépasser les capacités d'extraction de ces colonnes SPE et donc de sous-estimer les concentrations mesurées.

Ainsi l'apparition de nouvelles colonnes à support polymérique et non plus à support silice va remédier à tous ces inconvénients de la SPE que nous qualifierons de première génération.

Contrairement à l'extraction liquide-liquide qui utilise comme mode d'extraction le mode de partage, ces nouvelles colonnes SPE qui apparaissent sur le marché utilisent en dualité deux modes d' extraction : le mode de partage (phase inverse) et le mode d'échange d'ions. Ainsi, selon le pKa de la molécule à extraire et des conditions d'extraction ( $\mathrm{pH}$, choix tampon) le mode d'extraction prédominant sera différent. Par exemple, à $\mathrm{pH}$ basique, les analytes basiques sont décrochés du support de l'échangeur cationique alors qu'ils sont retenus sur ce support à $\mathrm{pH}$ acide. Le mode de partage participe au même temps au processus d'extraction de ces analytes basiques. Ainsi, on comprend mieux que ce double mode de rétention permet d'extraire plus de molécules avec un seule protocole et avec de meilleurs rendements comparé au mode d'extraction liquideliquide. Il est cependant important de dire que la technique d'extraction SPE est plus complémentaire que concurrentielle de l'extraction liquide-liquide et ceci pour une raison simple : dans notre pratique quotidienne, les spécimens biologiques à traiter sont divers et variés. Il est vrai que nous devrons disposer de plusieurs techniques d'extraction comme c'est déjà le cas pour les méthodes d'analyse.

Les inconvénients des méthodes d'extraction liquide- liquide sont nombreux : en premier lieu, elles ne sont pas automatisables ; en second lieu, la quantité de solvants utilisés est importante; et en dernier lieu, les extraits ne sont pas souvent propres. Ceci étant, les méthodes d'extraction liquide-liquide sont encore incontournables pour certaines extractions, d'autant plus que la toxicologie analytique moderne commence à traiter de plus en plus de matrices biologiques alternatives (bile, cheveux, humeur vitrée, méconium, etc.). Les colonnes SPE que nous avons testées sont différentes par la chimie de garnissage. Ceci a conduit à des taux d'extraction différents pour le protocole choisi. Par exemple, les colonnes Certify et Isolute $\mathrm{HCx}$ contiennent une résine échangeuse de cations forte ; au

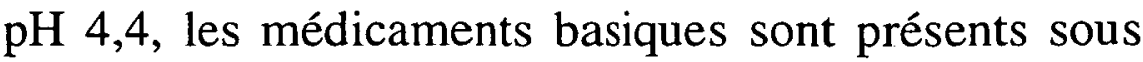
leur forme protonée et sont fortement retenus durant la phase d'élution par le méthanol. Ceci conduit obligatoirement à de faibles taux d'extraction pour ces produits. L'ajout de l'ammoniac dans la phase d'élution permet un recul d'ionisation de ces molécules et donc leur extraction aisée.

Pour le protocole d'extraction choisi la colonne OASIS semble, la colonne de choix pour l'extraction de divers toxiques médicamenteux dans le sérum et le sang total. Il est vrai que le double mode d'extraction de cette colonne permet d'extraire les molécule polaires et nonpolaires. Certify était parmi les premières colonnes à présenter une dualité de ce type d'extraction et cela incluait en plus des sites fonctionnels non-polaires (C8 dans le cas du Certify par exemple), des sites fonctionnels d'échanges de cations (SCX pour la colonne Certify par exemple). Ces différentes chimies permettent aussi l'utilisation de solvants organiques pour la phase de lavage des colonnes. Ainsi, la combinaison de ces deux mécanismes d'extraction permet de donner lieu à de larges extractions avec des extraits propres, ce qui permet secondairement d'améliorer la sensibilité analytique.

Certains auteurs comme Bogusz et Erkens (10) ont préféré l'utilisation d'une extraction liquide-liquide. La fraction acide est extraite par de l'éther éthylique à $\mathrm{pH}$ 4,6 et la fraction basique est extraite par un mélange de solvant dichlorométhane : isopropanol $(9: 1 \mathrm{v}: \mathrm{v})$ à $\mathrm{pH}$ 8,5. En général, beaucoup de laboratoires de Toxicologie utilisent le même schéma d'extraction avec des variantes pour le choix des solvants et des différences minimes pour le choix des $\mathrm{pH}$. Ce schéma d'extraction a été appliqué sur des colonnes à phases inertes (algues diatomées) comme les cartouches Extrelut (Merck) et Tox-Elut (Varian). L'avantage de ces phases inertes est la non-formation de mousse par 
contact de phases non miscibles et, en général, l'obtention d'extraits propres. Une technique d'extraction liquide-liquide prête à l'emploi est développée par la firme Toxi-Lab (Irvine, CA) et se présente sous forme de tubes appelés Toxi-tube $A$ et $B$ et sont commercialisés en France par la société Amilabo.

Chen et al (11) ont utilisé la colonne Certify afin d'extraire les fractions acides et basiques en deux étapes à partir des urines et du sérum. Leurs résultats se rapprochent des nôtres en utilisant la colonne OASIS. LAI et al (12) ont voulu, comme nous, faire une extraction en une seule étape sur la colonne Certify. Pour ce faire, ils ont modifié le protocole proposé par Chen et al (11) et ont utilisé comme solvant d'élution un mélange méthanol : ammoniac $(5: 1 \mathrm{v}: \mathrm{v})$. En détaillant leurs résultats, le taux d'extraction de $20 \mathrm{mg} / \mathrm{l}$ de salicylés par leur technique était nul. Pour leurs autres résultats, les taux d'extraction se rapprochent de ceux que nous avons obtenu avec la colonne OASIS. En ce qui concerne notre étude, seules les colonnes OASIS et MN HR-P ont permis l'extraction des salicylés.

De nouvelles colonnes SPE apparaissent sur le marché (Abselut de Varian, Oasis MCX de Waters). Ces dernières colonnes ont la particularité d'être mouillables et donc de pouvoir supprimer l'étape de conditionnement du processus d'extraction.

En conclusion, ce travail a montré l'intérêt des méthodes d'extraction SPE dans l'extraction d'un grand nombres de toxiques médicamenteux à partir de milieux biologiques comme le sérum ou le sang total. La colonne OASIS a permis des taux de rendements acceptables pour le protocole choisi avec une extraction en une seule étape de médicaments à chimies différentes. L'un des avantages de la SPE est la possibilité d'automatiser l'extraction. Un avantage futur de la SPE sera l'utilisation des volumes d'élution encore plus faibles ( 3 à 10 fois moins que ceux utilisés actuellement) grâce à la technologie des disques SPE et permettra de faibles prises d'essai. La technologie des 96 puits pour accélérer les cadences d'extraction est en plein essor actuellement, mais nous ne pensons pas que ce soit la priorité actuelle des laboratoires de Toxicologie analytique.

\section{Références}

1. Sadeg N., François G., Petit B., Dutertre-Catella H., Dumontet M. Automated liquid-chromatographic analyzer used for toxicology screening in a general hospital : 12 months' experience. Clin. Chem. 1997 ; 43 : 498-504.

2. Lillsunde P., Korte T. Comprehensive drug screening in urine using SPE and combined TLC and GC -MS identification. J. Anal. Toxicol. 1991 ; 15 : 71-81.

3. Turcant A., Premel-Cabic A., Cailleux A., Allain P. Toxicological screening of drugs by microbore high-performance liquid chromatography with photodiode-array detection and ultraviolet spectral library searches. Clin. Chem. $1991 ; 37: 1210-5$.

4. Puopolo P.R., Volpicelli S.A., Johnson D.K., Flood J.G. Emergency testing (detection, confirmation and quantification) of basic drugs in serum by liquid chromatography with photodiode array detection. Clin. Chem. 1991; 37 : 2124-30.

5. Wolff K., Sanderson Mb., Hay A.W.M. A rapid horizontal TLC method for detecting drug of abuse. Ann. Clin. Biochem. $1990 ; 27: 482-88$.

6. Binder S.R., Regalia M., Biaggi-Mc Eachem M., Mozhar M. Automated liquid chromatographic analysis of drugs in urine by on line sample clean up and isocratic multicolumn separation. J. Chromatogr. 1989 ; 473 : $325-41$.

7. Anonyme. Principes des réactions FPIA. In : Abbott Laboratoires, ed. Axsym System. Manuel technique volume 1. Laboratoires Abbott, 1996 ; 3.7-3.8.

8. Breiter J. Thin-layer chromatographic screening test for the detection of the abuse of drugs and pharmaceuticals. Kontakte. $1974 ; 3$ : 17-24.

9. Trinder P. Rapid determination of salicylate in biological fluids. Biochem. J. $1954 ; 301-303$.

10.Bogusz M., Erkens M. Influence of biological matrix on chromatographic behavior and detection of selected acidic, neutral, and basic drugs examined by means of a standardized HPLC- DAD system. J. Anal. Toxicol. $1995 ; 19: 49-55$.

11.Chen X.H., Wijsbeek J., Franke J.P., De Zeeuw R.A. A single column procedure on bond elut certify for systematic toxicological analysis of drugs in plasma and urine. J. Forensic Sci. $1992 ; 37: 61-71$.

12.Chi-Kong Lai, Lee T., Au K-M., Yan-Wo Chan A. Uniform solid-phase extraction procedure for toxicological drug screening in serum and urine by HPLC with photodiode - array detection. Clin. Chem. 1997 ; 43 : 31225. 